\title{
Optomechanical Piston Engines under Subharmonic Drives
}

\author{
Nur Aslan, Ali Ü. C. Hardal, Özgür E. Müstecaplığlu \\ Department of Physics, Koç University, Istanbul 34450, Turkey \\ naslan15@ku.edu.tr
}

\begin{abstract}
We consider a two stroke heat engine based on cavity quantum optomechanics. We investigate coherent dynamics of the mechanical mode and quantum statistics of the system with different driving profiles at subharmonic frequencies by solving the quantum optical master equation.
\end{abstract}

OCIS codes: $120.4880,270.0270,000.6880$

A quantum heat engine (QHE) is a machine that produces finite power when it undergoes conventional heat cycles described by the quantum first law of thermodynamics [1]. An intriguing QHE based on cavity quantum optomechanics has recently been proposed by [2]. An autonomous, multimode extension of the model has been considered as well [3]. There is a huge difference between the optical and mechanical frequencies in the system. Especially driving the system at high optical frequency to run an engine cycle makes the operation rather costly energetically.

In this work we consider a generic quantum optomechanical system. The Hamiltonian of the system is given by

$$
\hat{H}=\omega_{c} \hat{a}^{\dagger} \hat{a}+\omega_{m} \hat{b}^{\dagger} \hat{b}-g \hat{a}^{\dagger} \hat{a}\left(\hat{b}+\hat{b}^{\dagger}\right)
$$

where $\hat{a}\left(\hat{a}^{\dagger}\right)$ and $\hat{b}\left(\hat{b}^{\dagger}\right)$ are the annihilation (creation) operators of the optical and mechanical modes with resonance frequencies $\omega_{c}$ and $\omega_{m}$, respectively. We flag the optomechanical coupling strength with the coefficient $g$.

The system is coupled to two heat baths of temperatures $T_{h}, T_{c}\left(T_{h}>T_{c}\right)$ and undergoes a two stroke heat cycle. While the coupling to the bath of temperature $T_{c}$ is taken to be continuous, the interaction with the hot bath is considered as a time dependent external drive with a frequency of $\omega_{d}<\omega_{m}$.

We investigate the coherent dynamics and quantum statistics of the system. We specifically consider square and sinusoidal pulse profiles in subharmonic resonance frequencies. Photon statistics, mean energy, entropy of and power output from the system are analysed by using quantum optical master equation. We find that certain subharmonic drives can yield more efficient engine operation than resonant drives.

\section{References}

1. Tien D. Kieu, “The Second Law, Maxwell's Demon, and Work Derivable from Quantum Heat Engines", Phys. Rev. Lett. 93, 140403 (2004).

2. K. Zhang, F. Bariani, P. Meystre, "Quantum optomechanical heat engine", Phys. Rev. Lett. 112, 150602 (2014).

3. A. Mari, A. Farace, V. Giovannetti, "Quantum optomechanical piston engines powered by heat", J. Phys. B 48, 175501 (2015). 